TI 2000-041/2

Tinbergen Institute Discussion Paper

\title{
Analytic Decision Rules for Financial Stochastic Programs
}

Arjen H. Siegmann

André Lucas 
Tinbergen Institute

The Tinbergen Institute is the institute for economic research of the Erasmus Universiteit Rotterdam, Universiteit van Amsterdam and

Vrije Universiteit Amsterdam.

Tinbergen I nstitute Amsterdam

Keizersgracht 482

1017 EG Amsterdam

The Netherlands

Tel.: +31.(0)20.5513500

Fax: $\quad+31 .(0) 20.5513555$

Tinbergen I nstitute Rotterdam

Burg. Oudlaan 50

3062 PA Rotterdam

The Netherlands

Tel.: $\quad+31 .(0) 10.4088900$

Fax: $\quad+31 .(0) 10.4089031$

Most TI discussion papers can be downloaded at

http://www.tinbergen.nl 


\title{
Analytic Decision Rules for Financial Stochastic Programs*
}

\author{
Arjen H. Siegmann ${ }^{\dagger \ddagger} \quad \bullet \quad$ André Lucas $^{\dagger \ddagger}$
}

This version: May 1, 2000

\begin{abstract}
Ontemporary financial stochastic programs typically involve a trade-off $\checkmark$ between return and (downside)-risk. Using stochastic programming we characterize analytically (rather than numerically) the optimal decisions that follow from characteristic single-stage and multi-stage versions of such programs. The solutions are presented in the form of decision rules with a clear-cut economic interpretation. This facilitates transparency and ease of communication with decision makers. The optimal decision rules exhibit switching behavior in terms of relevant state variables like the assets to liabilities ratio. We find that the model can be tuned easily using Value-at-Risk (VaR) related benchmarks. In the multi-stage setting, we formally prove that the optimal solution consists of a sequence of myopic (single-stage) decisions with risk-aversion increasing over time. The optimal decision rules in the dynamic setting therefore exhibit identical features as in the static context.
\end{abstract}

Keywords: downside-risk, stochastic programming, asset allocation, valueat-risk, time diversification, asset/liability management.

${ }^{*}$ We thank Guus Boender for useful discussions. André Lucas also thanks the Dutch Organization for Scientific Research (N.W.O.) for financial support. Correspondence to: siegmann@tinbergen.nl or alucas@econ.vu.nl.

${ }^{\dagger}$ Dept. Finance and Financial Sector Management, Vrije Universiteit, De Boelelaan 1105, NL-1081HV Amsterdam, the Netherlands. Phone: +31-(0)20-444 6034. Fax: +31-(0)20-444 6020.

${ }_{\ddagger}^{\ddagger}$ Tinbergen Institute Amsterdam, Keizersgracht 482, NL-1017EG Amsterdam, the Netherlands. 


\section{Introduction}

Over the past decade, we have witnessed a growing literature on financial planning models. Such models can assist financial institutions like pension funds, insurance companies and banks in their Asset/Liability Management (ALM), as illustrated in the book by Ziemba and Mulvey (1998). The key component in these models concerns the trade-off between risk and return. It is therefore of paramount importance which risk measure is put into the model. Traditionally, the variance or standard deviation has been the prominent measure of risk. Its main (and perhaps only) advantage is its computational simplicity. As argued by Sortino and Van der Meer (1991) however, the variance is an inadequate measure of risk in many practical circumstances. The main criticism to the use of the variance is its symmetric nature, whereas risk is typically perceived as an asymmetric phenomenon, see Kahneman and Tversky (1979). Asymmetric or downside risk measures are generally more difficult to work with. Given the current state of computer technology, however, their use in both theoretical and empirical financial planning models has increased rapidly. Note that, just as the variance, down-side risk measures can be embedded in an expected utility framework, see Fishburn (1977).

Downside-risk measures are currently used extensively in the area of Asset/Liability Management (ALM). Recent research typically incorporates the down-side risk measure in a multi-stage stochastic programming (MSP) approach, as in Consigli and Dempster (1998), Mulvey and Thorlacius (1998), Cariño et al. (1994), Boender (1997), and Dert (1998). The main advantage of the MSP approach relative to the more traditional static mean-variance oriented approach is that the explicit dynamic nature of financial decisions can better be taken into account. For example, a decision now may be followed by recourse actions in the future. Moreover, different preferences and (dynamic) constraints can be modeled directly. As a result, the MSP approach generally produces significant improvements over static mean-variance based decisions. These improvements can be exploited when the MSP model is implemented in practice, as in Cariño et al. (1994). The MSP-models used for ALM, however, also have two interrelated drawbacks: (i) the 
models are computationally intensive and difficult to solve, and (ii) the resulting optimal solution may be difficult to analyze and interpret. Generally, it is impossible to solve an MSP analytically. As an alternative, most people seek for computational solutions built around discretizations of the original MSP. Such discretizations typically involve the construction of a scenario structure that adequately captures the original uncertainty in the model. The MSP is then transformed into a deterministic equivalent program based on the scenario structure and solved numerically. The numerical solution is taken as an approximation to the solution of the original MSP. The computational effort needed to solve the deterministic programming problem increases rapidly in the number of scenarios used. Also, the numerical solution typically consists of optimal decisions at every point in time and in every possible state of nature (as represented in the scenario tree). Even for simple MSP models and realistically sized scenario structures, this gives an unwieldy set of numbers that lacks transparency and clear-cut economic interpretation. Moreover, the numerical solution is only optimal for the specific instance of the scenario tree, parameter values and initial state variables for which the problem is solved. See also Dupačová et al. (1998) who check the robustness of the optimal solution to a stochastic program with respect to out-of-sample scenarios. Consigli and Dempster (1998) note on the complexity of the solution to an MSP that "The solution to these very large and complex problems needs to be followed by a detailed computer-based analysis of the results in order to supply conveniently represented information to the decision maker." In other words, for these models to be implemented and used by management or decision makers, there is a need for a method to summarize optimal decisions in terms of decision rules, linking optimal decisions in each state of nature to observed quantities like the assets to liabilities ratio, the state of the economy, etc.

Some numerical attempts at optimizing over decision rules directly rather than over a set of unconstrained control variables are Berger and Mulvey (1998) and Boender (1997). Also Maranas et al. (1997) use a decision rule approach, but constrain the set of rules to a fixed-mix. 
In some continuous-time models, optimal parametric decision rules can be derived analytically. Some examples are Merton (1969) and Sethi (1998) for the consumption/investment problem and Ingersoll (1987) for the general portfolio optimization problem. In a recent paper, Basak and Shapiro (1999) optimize expected utility of terminal wealth under a constraint on downside-risk. To derive analytic decision rules however, they have to assume normality and complete markets, which is unrealistic in practice.

Our main contribution in the present paper is that we analytically characterize optimal decision rules for a typical financial planning problem modeled as an MSP model. In contrast to the continuous-time approach of Basak and Shapiro (1999), we derive results in discrete time without using the assumptions of complete markets or normality. Instead we work with incomplete markets and leave the distributional properties of the model unspecified. We concentrate on a model where expected wealth is traded off against expected loss (or shortfall) with respect to a certain benchmark level. As such, it is prototypical for many financial planning models used in practice. Similar models have been studied by Cariño et al. (1994), Hiller and Eckstein (1993) and Uryasev and Rockafellar (1999) from a numerical rather than an analytical perspective. We study both a static (one-period) and dynamic (multi-stage) version of the model. By specifying the solutions in the form of decision rules, we provide insight into the crucial mechanisms driving the optimal decisions.

For the one-period model we find that the optimal amount invested in the risky asset increases linearly in the investor's surplus, i.e. in the difference between current wealth and the benchmark level, if the surplus is positive. A decreasing linear relation is found for negative surplus values. This induces risk taking by the manager in both favorable and unfavorable positions. In unfavorable situations (negative surplus) risk taking is the only way in which recovery can be established. In favorable circumstances, investing in the risky asset increases future expected wealth without unduely increasing downsiderisk. A straightforward implication of this result is that the fraction invested in the risky asset is a non-monotonic and non-linear function of the wealth-to-benchmark (or assets 
to liabilities) ratio.

The decision rule approach facilitates the adoption of MSP models by financial managers. The only remaining difficulty is that the manager has to specify her trade-off between expected wealth and expected loss/shortfall. To facilitate this trade-off, we link our model parameters to the choice of a Value-at-Risk(VaR) percentage. VaR is the maximum loss over a given horizon for a given confidence level. Although Artzner et al. (1999) argue that Value-at-Risk is not a coherent risk measure, it is nowadays widely used and accepted in the financial industry. Therefore, whereas it may be difficult for managers to formulate criteria for the expected amount of shortfall, it may be much easier to postulate bounds on the acceptable probability of shortfall. In this way the popularity of VaR might actually contribute to a further adoption of models that incorporate expected loss as a risk measure, as in our model. Interestingly, the link with VaR further aligns the optimal solution with economic common sense. In particular, the switch from safe to risky investments for (increasingly) low wealth can be made much more gradual in a natural way. This is a desirable feature as highly risky investment strategies in unfavorable situations are generally not accepted as a sound policy in an empirical context.

For the multi-stage version of our model we find the same typical shape of the optimal decision rules as for the one-period model. Moreover, the multi-stage model can be solved as a sequence of "almost" myopic one-period problems. As the risk aversion parameter associated with these one-period problems decreases over time, we find that the steepness of the decision rules increases in the time to maturity. This result corroborates the idea of time-diversification, which states that investors will hold riskier portfolios whenever the planning period is longer.

The remainder of the paper is set up as follows. For ease of exposition, we start with a one-period model in Section 2. For this model, we derive the decision rules and give the relation between Value-at-Risk preferences and model parameters. Section 3 gives the multi-stage version of the same model and the corresponding optimal decision rules. Concluding remarks are given in Section 4. The Appendix gathers the proofs. 


\section{The one-period model}

\subsection{Model setup}

Consider an investor who has to decide on her investment strategy with initial wealth $W_{0}$. We assume that the investor maximizes expected wealth $W_{1}$ at time 1 . Risk is perceived as the difference between terminal wealth and a certain benchmark level $W_{1}^{B}$. For example, if $W_{1}^{B}=W_{0}$, terminal wealth is measured against initial wealth and interpreted in terms of profits and losses. The target $W_{1}^{B}$ can be interpreted as an internal target or as an externally given benchmark. As a typical example, for a pension fund $W_{1}^{B}$ can be interpreted as the fund's liabilities.

For simplicity, we only consider two assets. The first is a risk-free asset, yielding a constant rate of return of $r_{f}$ (e.g. 1.04). The second is a risky asset with an uncertain return $u$, e.g. the return on stocks. We assume that $u$ has a known absolute-continuous* distribution function $G(\cdot)$ with support $(0, \infty)$ and $\mathbb{E}[u]>r_{f}$. Defining $X_{0}$ as the initial amount invested in the risky asset, we have

$$
W_{1}=W_{0} r_{f}+X_{0}\left(u-r_{f}\right)
$$

with $W_{1}$ denoting the value of assets at time 1 . We postulate the following objective function for the one-period optimization problem:

$$
\left.\max _{X_{0}} \mathbb{E}_{G}\left[W_{1}\right)\right]-\lambda \cdot \mathbb{E}_{G}\left[W_{1}^{B}-W_{1}\right]^{+}
$$

with $\lambda>0, \mathbb{E}_{G}(\cdot)$ denoting expectation with respect to $G$, and $[y]^{+}$denoting the maximum of 0 and $y$. The first term in (2) gives expected final wealth and is increasing in $X_{0}$. The second term gives the measure of risk, namely expected shortfall. According to Artzner et al. (1999) expected shortfall is a coherent risk measure, as opposed to the popular Value-at-Risk measure. Fishburn (1977) shows that with (2) as the utility function the

\footnotetext{
* Our results also extend to the case of discrete distribution functions $G(\cdot)$, but proving this requires substantial additional mathematical effort without providing additional insights.
} 
mean-risk utility model with risk defined by expected loss is congruent with the expected utility model. Hiller and Eckstein (1993) use exactly the same objective function, and propose this model as a stochastic dedication model for fixed-income portfolios. The risk measure in (2) differs only with that of Cariño et al. (1994) in that they use a piecewise linear penalty function in terms of the expected loss, where we have a linear one, as determined by $\lambda$. Cariño et al. (1994) also illustrate that in the context of banks and insurance companies risk measures as in (2) can easily be justified. For these institutions there are specific costs associated with the situation of cash reserves falling below a certain critical level. Note that expected shortfall/loss is also used by Basak and Shapiro (1999) who include it in a constraint in the context of a (Gaussian) continuous-time dynamic portfolio optimization model.

The risk aversion parameter $\lambda$ in (2) determines the trade-off between risk and return. Setting $\lambda=0$ implies risk neutrality, while increasing $\lambda$ induces increases in risk aversion. For the moment, we assume $\lambda$ is determined directly by the decision maker. At the end of this section though, we show that $\lambda$ can be set directly by specifying VaR preferences.

Finally, note that model (2) is a one-period optimization problem, while the MSPmodels found in the literature are multi-stage and dynamic. In Section 3 we show that the optimal solution to (2) has exactly the same form as that of a dynamic multi-stage model.

\subsection{Solution to the one-period model}

For ease of exposition we define the one period discounted benchmark wealth $W_{0}^{B}$ as $W_{1}^{B} / r_{f}$. Furthermore, we define the time zero surplus $S_{0}$ as $W_{0}-W_{0}^{B}$, which is the amount of current wealth exceeding the time zero required level $W_{0}^{B}$. Note that in an asset-liability management framework, $W_{0}^{B}$ can be interpreted as the (discounted) liabilities such that the surplus $S_{0}=W_{0}-W_{0}^{B}$ has its natural interpretation as assets minus liabilities. 
Lemma 2.1 Consider the special case $W_{0}=W_{0}^{B}$. If the following condition holds

$$
\int_{u=0}^{r_{f}}\left(r_{f}-u\right) d G>\frac{\mathbb{E}_{G}\left[u-r_{f}\right]}{\lambda}
$$

then the optimal solution to (2) is $X_{0}^{*}=0$. Otherwise, $X_{0}^{*}$ is infinite.

Proof: See appendix

As we want to ensure that a finite solution to (2) exists in case $W_{0}=W_{0}^{B}$, we require that condition (3) holds for any $\lambda$. It puts a lower bound on the risk aversion $\lambda$ for a given distribution $G(\cdot)$ and risk-free rate $r_{f}$.

The main results for the one-period model follow from the following lemma

Lemma 2.2 The first order condition to (2) is given by

$$
\int_{0}^{\bar{u}}\left(r_{f}-u\right) d G=\frac{\mathbb{E}_{G}\left[u-r_{f}\right]}{\lambda}
$$

where $\bar{u}$ is defined as

$$
\bar{u}=\frac{W_{1}^{B}-W_{0} r_{f}}{X_{0}}+r_{f}
$$

Moreover, the optimal $X_{0}$ is nonnegative.

Proof: See appendix

The consequence of lemma 2.2 is that solving (2) is equivalent to finding the return $\bar{u}$ that solves (4). As there is a one-to-one correspondence between a value of $\bar{u}$ and that of $X_{0}$, the corresponding $X_{0}^{*}$ then follows from relation (5) and the condition $X_{0}^{*}>0$. Given condition (3), it turns out that there are two distinct values $\bar{u}^{*}$ that satisfy (4). This is depicted in figure 1 for a lognormal distribution. The integral on the left-hand side of (4) is drawn as a function of the upper bound $\bar{u}$. The right-hand side of (4) is drawn as the horizontal line at $\mathbb{E}\left[u-r_{f}\right] / \lambda$.

The focus of this paper is on the optimal solution $X_{0}^{*}$ in terms of the unknown $\bar{u}$. The next theorem presents the main results for the one-period model. 


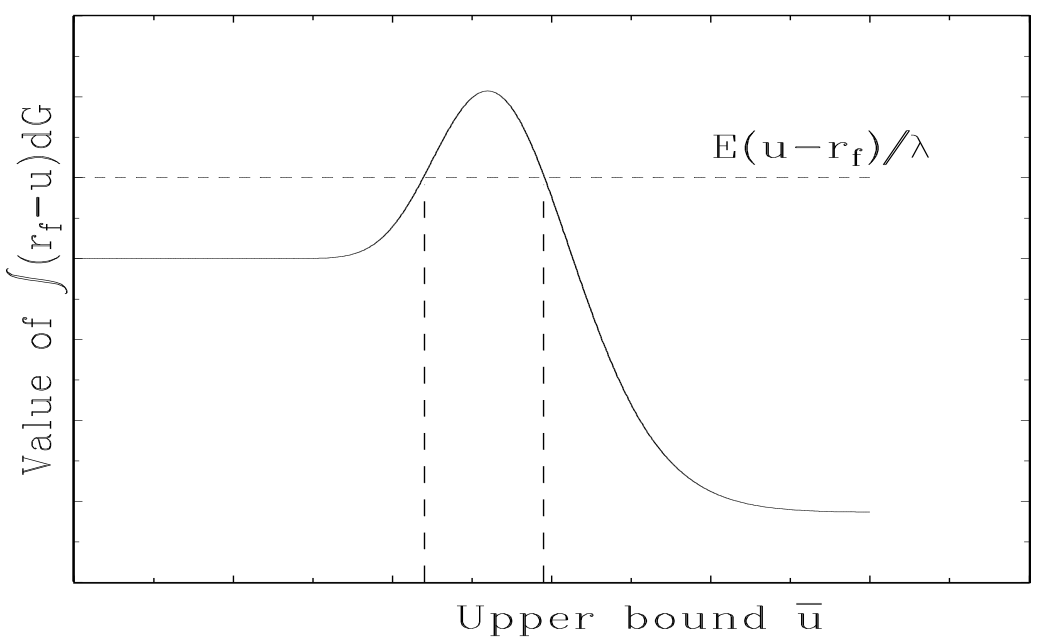

Figure 1: In this figure the solid line represents the value of the integral of the left-hand side of (4) as a function of $\bar{u}$ with $G$ lognormal. The horizontal dashed line shows the constant term at the right hand side of (4)

Theorem 2.1 If condition (3) is satisfied, for the case $W_{0}>W_{0}^{B}$ there is a $\bar{u}^{+}<r_{f}$ that solves the first order condition (4), resulting in the following optimal decision rule for $X_{0}$ :

$$
X_{0}^{*}=\frac{r_{f}}{r_{f}-\bar{u}^{+}} \cdot\left(W_{0}-W_{0}^{B}\right) .
$$

For the case $W_{0}<W_{0}^{B}$ there is a $\bar{u}^{-}>r_{f}$ that solves the first order condition (4), giving:

$$
X_{0}^{*}=\frac{r_{f}}{\bar{u}^{-}-r_{f}} \cdot\left(W_{0}^{B}-W_{0}\right)
$$

Proof: See appendix.

Theorem 2.1 shows that for a positive surplus $W_{0}-W_{0}^{B}$ the decision $X_{0}^{*}$ has a positive linear relation with the surplus. In case of a negative surplus the relationship is negative and linear. The first remarkable consequence of Theorem 2.1 is that the piecewise linearity in the optimal decision rule for $X_{0}$ holds for any continuous distribution function $G(\cdot)$ satisfying the conditions of Subsection 2.1. The distribution of the uncertain return $u$ 
only enters the optimal decision rule through the values of $\bar{u}^{+}$and $\bar{u}^{-}$, which follow from equation (4). An economic interpretation for the optimal values of $\bar{u}$ is presented in Subsection 2.3, together with the issue of picking $\lambda$ in a practical situation. Also note that condition (3) is needed again to ensure that the optimal $X_{0}$ is finite, see the proof in the appendix.

The second consequence of Theorem 2.1 follows from the two different decision rules for $X_{0}^{*}$. The amount invested in the risky asset is decreasing in the surplus when the surplus is negative, and increasing in case it is positive. This typical behavior of the optimal decision rule implies that in an unfavorable situation (negative surplus) the decision maker takes more risk as the surplus decreases. In the favorable situation of a positive surplus, the decision maker takes more risk when the surplus increases. The fact that the slope of the decision rule is different for the two regimes is due to the different risk taking behavior. In a situation of a negative surplus the decision maker must take more risk: the higher return on the portfolio is needed to make the probability of recovering from the unfavorable situation strictly positive. In the situation of a positive surplus risky investments allow one to profit from the higher expected return, subject to having an acceptable trade-off with downside-risk. Clearly, if the surplus is positive and wealth increases, more money can be invested in the risky asset at the cost of a marginal increase only in downside risk. This induces a positive relation between the surplus and the investment in the risky asset.

The third implication of Theorem 2.1 is obtained by switching from amounts to ratios. The motivation for this change is that it is quite common to present investment policies in terms of the fraction invested in stocks. It eases the comparison between different investors (that might have different absolute wealth levels) and at the same time enhances the communication with management. In our model, the transformation is established by dividing the left-hand and right-hand side of (6) and (7) by $W_{0}$. The result is given by the following corollary: 
Corollary 2.1 The optimal fraction invested in the risky asset is given by

$$
\left(\frac{X_{0}}{W_{0}}\right)^{*}=\frac{r_{f}}{\bar{u}^{*}-r_{f}} \cdot\left(1-\frac{W_{0}^{B}}{W_{0}}\right),
$$

where $\bar{u}^{*}$ is equal to $\bar{u}^{+}$or $\bar{u}^{-}$as in Theorem 2.1, depending on the sign of the surplus $W_{0}-W_{0}^{B}$.

The simple transformation from amounts to ratios gives a remarkable result. With $\widehat{W}_{0}=W_{0} / W_{0}^{B}$, Figure 2 displays the typical shape of the relation between $\widehat{W}_{0}$ and the fraction in the risky asset. As mentioned earlier, in an asset-liability management framework $\widehat{W}_{0}$ can be interpreted as the assets to liabilities or funding ratio, which is one of the key variables in that context. We find that for low ratios $\widehat{W}_{0}$ the relation is convex, while for high $\widehat{W}_{0}$ it is concave. The two regimes (low versus high funding ratio) follow from the two different values $\bar{u}^{+}$and $\bar{u}^{-}$which solve (4). The typical shapes illustrate the different risk taking behavior for a favorable versus an unfavorable situation, as discussed above.

\subsection{Choosing the preference parameter $\lambda$}

We have now solved the one-period financial planning model for a given value of $\lambda$. As argued earlier, it may not be easy for a decision maker to choose a specific value of $\lambda$ that adequately reflects her risk-return profile. This is mainly due to the fact that $\lambda$ does not have a direct interpretation in practical asset management. Decision makers rather have an opinion on practical concepts like the tolerable Value-at-Risk (VaR) or loss probability than on $\lambda$. In this section, we show that $\lambda$ can be set unambiguously on the basis of VaR related preferences.

We distinguish between two situations: (i) a positive surplus and (ii) a negative surplus. First, we concentrate on case (i). From Figure 1 and the discussion in the previous subsection we note that a given $\lambda$ (combined with a positive surplus) results in an op-

timal $\bar{u}^{+}<r_{f}$. This argument can be reversed: a given value of $\bar{u}^{+}<r_{f}$ gives rise to a corresponding value of $\lambda$. So instead of picking $\lambda$, we can also set the value of $\bar{u}^{+}$. 


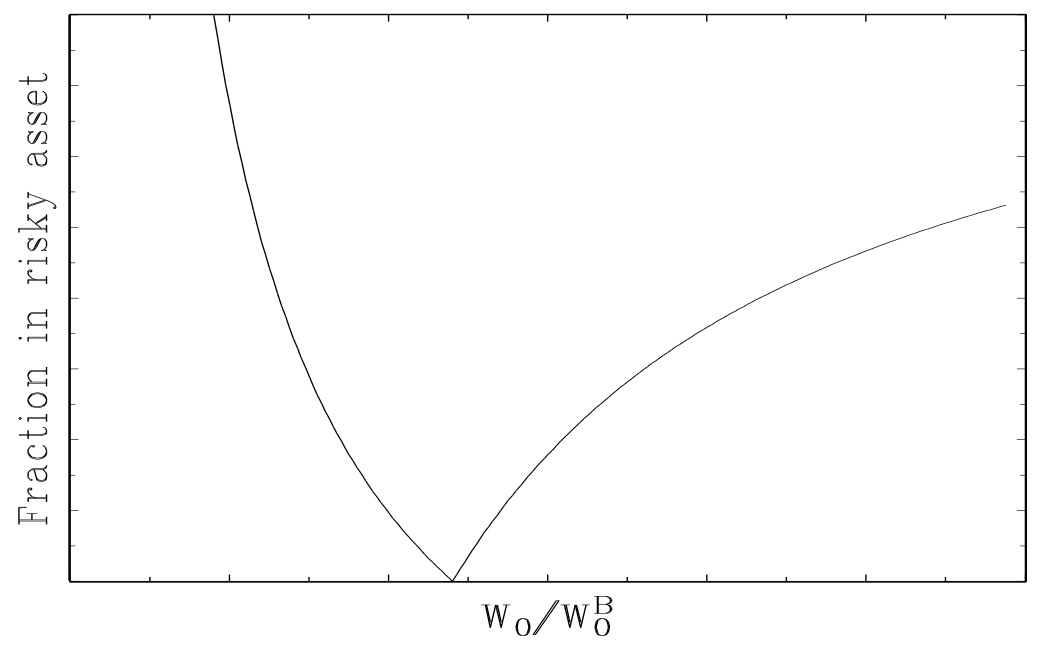

Figure 2: The typical shape of the relation between the optimal fraction in the risky asset and the funding ratio. The convex (left) part of the graph gives the decision rule in case of a negative surplus. The concave (right) part of the graph is valid for a positive surplus.

The advantage setting $\bar{u}^{+}$rather than $\lambda$ is that $\bar{u}^{+}$has a more direct economic interpretation. In particular, $\bar{u}^{+}$gives a critical realization of the return on the risky asset: for returns $u_{1}<\bar{u}^{+}$, a negative surplus results, whereas a positive surplus emerges for returns $u_{1}>\bar{u}^{+}$. We now assume that the manager sets the maximum tolerable probability of a negative surplus. This amounts to the manager choosing a specific $\alpha \in(0,1)$, such that $\operatorname{Pr}($ Surplus $<0)=\alpha$. Note again that it may be much easier for managers to set $\alpha$ than to set $\lambda$, as for given $\alpha$, the level $W_{0}^{B}$ can be interpreted as the $\alpha$-level VaR. As a negative surplus only occurs for $u_{1}<\bar{u}^{+}$, we have $\alpha=G\left(\bar{u}^{+}\right)$or $\bar{u}^{+}=G^{-1}(\alpha)$. Note that $\alpha$ must be such that $G^{-1}(\alpha)<r_{f}$ in order to satisfy condition (3). From $G^{-1}(\alpha)$ we can then derive the corresponding value of $\lambda$ from (4). Note that setting $\alpha$ instead of $\lambda$ has the additional advantage that we only have to solve the first order condition (4) if the precise numerical value of $\lambda$ is required. Otherwise, we only have to invert the $\operatorname{cdf} G(\cdot)$ and plug the desired $\bar{u}^{+}=G^{-1}(\alpha)$ into (5). 
By relating surplus management as in (2) to VaR objectives, it thus appears that managing expected loss (indirectly) leads to VaR management. Similar results can be found in Uryasev and Rockafellar (1999) and Basak and Shapiro (1999).

The same approach can be followed if the surplus $W_{0}-W_{0}^{B}$ is negative. In that case, however, it is more natural to interpret $\alpha$ as a non-recovery probability rather than a VaR probability. For example, in case of underfunding the manager might want a recovery probability of $75 \%$, meaning that she would like to end up with a positive surplus in $75 \%$ of the cases. We then set $\alpha=0.25=G\left(\bar{u}^{-}\right)$and solve for the corresponding value of $\bar{u}^{-}$. Note again that $\alpha$ has to be chosen such that $\bar{u}^{-}=G^{-1}(\alpha)>r_{f}$. The distinction between positive and negative surplus and the corresponding use of VaR and recovery probabilities, respectively, allows us to model different risk attitudes in different financial circumstances. Such additional flexibility enhances the practical implementability of our model. Moreover, tuning on $\mathrm{VaR}$ and recovery probabilities rather than on $\lambda$ results in reduced computational complexity as well as better managerial interpretability.

To give some intuition for the different shapes of the decision rules, we plot the optimal asset allocation $X_{0}^{*}$ as a function of the wealth to benchmark ratio for varying VaR and recovery probabilities. The results are in Figure 3.

We see that a higher VaR or recovery probability results in more aggressive strategies: the optimal asset mix switches more rapidly from safe to risky assets for varying wealth to benchmark ratios. Moreover, the aggressiveness of the resulting strategy can be linked directly to the manager's VaR and recovery preferences. We also note that by setting the VaR probability different from the recovery probability, we can separately tune the steepness of $X_{0}^{*}$ as a function of $W_{0} / W_{0}^{B}$ for $W_{0}$ smaller and larger than $W_{0}^{B}$, respectively. So if the optimal strategy for a negative initial surplus is deemed too aggressive, the recovery probability can be decreased until the solution becomes acceptable. 


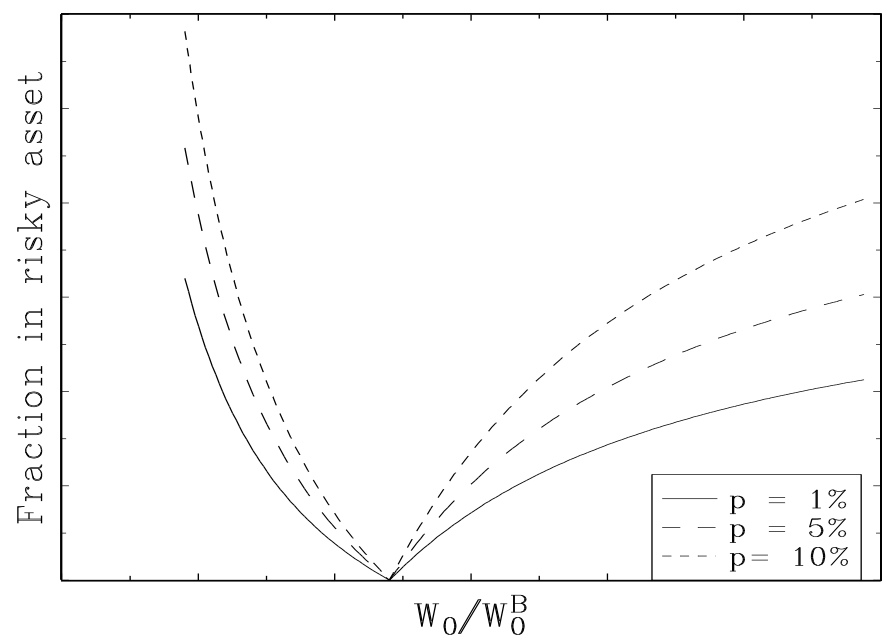

Figure 3: The decision rules for $X_{0}^{*}$ for different recovery and shortfall probabilities. At the left side of the graph, which represents the decision rule in case of a negative surplus, $p$ represents the recovery probability, i.e., the probability of attaining wealth above the benchmark level $W_{1}^{B}$. At the right-hand side of the graph, $p$ is the probability of shortfall, i.e. the probability of final wealth falling below $W_{1}^{B}$. 


\section{The multi-stage model}

We now turn to the multi-stage generalization of (2) and obtain four main results. First, the dynamic model can be solved as a sequence of one-period models, each one of the same form as that in Section 2. Second, as a the direct consequence, the decision rule for each optimal decision at time $i$ is of the same form as that for the one-period optimal decision $X_{0}^{*}$. Third, we prove that the sensitivity of the decision rules to changes in (relative) wealth increases when the horizon extends. Finally, we show that there is again a unique VaR-quantile associated with a certain value of $\lambda$. This allows for the same way of tuning the model as discussed in Subsection 2.3 for the one-period model.

The $n$-stage model is optimized over a sequence of decisions $X_{0}, X_{1}, \ldots, X_{n-1}$. The model is dynamic in the sense that every $X_{i}$ is chosen contingent on the information at time $i$. The objective function is defined in terms of shortfall and wealth at the end of the final period,

$$
\begin{array}{cl}
\underset{X_{0}, \ldots, X_{n-1}}{\max } & \mathbb{E}\left[W_{n}\right]-\lambda \cdot \mathbb{E}_{G}\left[W_{n}^{B}-W_{n}\right]^{+}, \\
\text {s.t. } & W_{i+1}=W_{i} r_{f}+X_{i}\left(u_{i+1}-r_{f}\right), \quad i=1, \ldots, n,
\end{array}
$$

with $u_{i+1}$ the return on the risky asset over period $i+1$ and $\lambda>0$. The returns are assumed to be independent, though not necessarily identically distributed.

We define $W_{j}^{B}=W_{n}^{B} / r_{f}^{n-j}$ as the riskfree discounted value of benchmark wealth at time $j$. The main results for the multistage model follow from the following theorem.

Theorem 3.1 In the multi-stage model with $n$ stages, the first order condition with respect to $X_{i-1}$ is

$$
p_{i}^{-} \cdot \int_{0}^{\bar{u}_{i}}\left(r_{f}-u_{i}\right) d G_{i}\left(u_{i}\right)+p_{i}^{+} \cdot \int_{\bar{u}_{i}}^{\infty}\left(r_{f}-u_{i}\right) d G_{i}\left(u_{i}\right)-\mathbb{E}\left[u_{i}-r_{f}\right] / \lambda=0,
$$

where the $\bar{u}_{i}$ are defined as

$$
\bar{u}_{i}=r_{f}+\frac{W_{i}^{B}-W_{i-1} r_{f}}{X_{i-1}}
$$


and the $p_{i}^{+}$and $p_{i}^{-}$are recursively defined by

$$
\begin{aligned}
p_{i}^{-} & =p_{i+1}^{-} \cdot G_{i}\left(\bar{u}_{i}^{-}\right)+p_{i+1}^{+}\left(1-G_{i}\left(\bar{u}_{i}^{-}\right)\right), \\
p_{i}^{+} & =p_{i+1}^{-} \cdot G_{i}\left(\bar{u}_{i}^{+}\right)+p_{i+1}^{+}\left(1-G_{i}\left(\bar{u}_{i}^{+}\right)\right),
\end{aligned}
$$

and $p_{n}^{-}=1, p_{n}^{+}=0$.

Proof: See appendix.

We can rewrite the first order condition in (11) as

$$
\int_{0}^{\bar{u}_{i}}\left(r_{f}-u_{i}\right) d G_{i}\left(u_{i}\right)=\lambda_{i}^{-1} \cdot \mathbb{E}\left[u_{i}-r_{f}\right]
$$

where

$$
\lambda_{i}^{-1} \equiv \frac{1 / \lambda+p_{i}^{+}}{p_{i}^{-}-p_{i}^{+}}, \quad i=1, \ldots, n,
$$

By comparing (15) with (4), we find that the first order condition with respect to $X_{i-1}$ in the $n$-stage model is of the same form as the first order condition in the one-period model. Hence, the decision rule will be the same, differing only because $\lambda$ in the one-period model is replaced by $\lambda_{i}$ and $G(\cdot)$ is replaced by $G_{i}(\cdot)$.

Before we present the decision rule, note that in line with condition (3) in Subsection 2.1 we require

$$
\int_{0}^{r_{f}}\left(r_{f}-u_{i}\right) d G_{i}<\lambda_{i}^{-1} \cdot \mathbb{E}\left[u_{i}-r_{f}\right], \quad i=1, \ldots, n
$$

in order to ensure a finite optimal solution. Condition (17) at the same time ensures that the optimal decision for $W_{i}=W_{i}^{B}$ is $X_{i}^{*}=0$.

We have the following corollary.

Corollary 3.1 Subject to (17) the solution to the optimization problem in (9) and (10) can be characterized as

$$
X_{i}^{*}=\frac{r_{f}}{\bar{u}_{i+1}^{*}-r_{f}} \cdot\left(W_{i}^{B}-W_{i}\right), \quad i=0, \ldots, n-1,
$$


where $\bar{u}_{i+1}^{*}$ is either equal to $\bar{u}_{i+1}^{+}<r_{f}$ or $\bar{u}_{i+1}^{-}>r_{f}$ for $S_{i}>0$ or $S_{i}<0$, respectively. Moreover, the $\bar{u}_{i+1}^{*}, i=1, \ldots, n$, are fixed constants.

Proof: See appendix.

Corollary 3.1 shows that the optimal decision rules are the same as the typical piecewiselinear optimal rules for the one-period model.

Another remarkable implication of Theorem 3.1 is that the $n$-stage model can be solved by solving a sequence of one-period problems. Using (15) for $i=n$ gives $\bar{u}_{n}^{-}$and $\bar{u}_{n}^{+}$. Substituting these in (13), (14) and (16) gives $\bar{u}_{n-1}^{-}$and $\bar{u}_{n-1}^{+}$from (15) again. The optimal policies are given by (18) and the algorithm continues until we have found the decision rule for $X_{0}^{*}$. In fact, we have found a decomposition which makes it possible to solve the MSP quite easily. We only have to solve $(2 \times n)$ one-period models to characterize the complete solution in terms of the optimal decision rules instead of having to solve one large multi-stage model. Moreover, as (15) is easily solved numerically for given $\lambda_{i}$ and $G_{i}(\cdot)$, it is possible to numerically solve the dynamic program without resorting to scenarios and deterministic equivalents of the original MSP.

The following corollaries give an important property of the $\lambda_{i}$ 's and its implication for the optimal investment strategies, respectively.

Corollary 3.2 The values of $\lambda_{i}^{-1}$ are increasing in the time to maturity $n-i$.

Proof: See appendix.

Corollary 3.3 If the $u_{i}$ are identically distributed, the absolute slope $r_{f} /\left|u_{i}^{*}-r_{f}\right|$ of the optimal decision rules in (18) is monotonically increasing in the time to maturity $n-i$.

Proof: See appendix. 
As the $\lambda_{i}^{-1} \mathrm{~s}$ are the inverse of the degree of risk aversion, Corollary 3.2 states that the degree of risk aversion $\left(\lambda_{i}\right)$ in the multi-stage setting decreases with the time to maturity. As a direct result, Corollary 3.3 concludes that the optimal policies are more sensitive to changes in surplus if the planning horizon is further away. As for a zero initial surplus the investment in the risky asset is zero, independent of the length of the planning period, the increaseing slope of the decision rules implies riskier intial asset allocations for longer planning periods and given surplus.

Interestingly, Corollaries 3.2 and 3.3 also have implications in the area of time diversification theory. Proponents of time diversification argue that investors will hold a riskier asset allocation whenever the investment horizon is further away. This is based on the observation that in the long run stock returns almost certainly outperform bond returns. However, it is argued by Samuelson (1994), Kritzman and Rich (1998) and Merton and Samuelson (1974) that the spread of the distribution of terminal wealth also widens with the time horizon. Consequently, the potential magnitude of the loss increases accordingly if riskier investments are held. This is used as an argument to prove that it could well be that an investor chooses a less aggressive portfolio if the investment horizon increases. Corollary 3.3 proves, however, that if wealth is measured against a benchmark level, and the magnitude of a loss is taken into account, under a dynamic policy risk averse investors will hold a riskier asset allocation whenever the horizon is longer. Note that if we take $W_{n}^{B}=0$, time-diversification also holds for the absolute wealth level $W_{i}$.

Finally, the following corollary ensures that the multi-stage model can also be tuned appropriately by relating the model parameters to VaR related benchmarks.

Corollary 3.4 In initial surplus is positive, there is a unique $\lambda^{+}$associated with the choice of a VaR-quantile. For a negative surplus, there is a unique $\lambda^{-}$associated with a recovery probability.

Proof: See appendix. 
Corolloray (3.4) implies that the decision maker does not have to pick $\lambda$ in the multistage model directly. As the probabilities of a positive and a negative final surplus are uniquely determined by a choice of $\lambda$, the manager only has to specify her VaR-preferences and a value for $\lambda$ follows immediately. Of course the choice for a VaR-quantile is again limited by condition (17), ensuring that the optimal decisions $X_{i}^{*}$ are finite.

\section{Conclusions and discussion}

In this paper we analytically solved a typical financial planning model involving a tradeoff between return and downside-risk. Our model shares the same basic characteristics of more elaborate empirical models like that of Cariño et al. (1994). Downside-risk was used because of its widespread popularity in the financial industry and its use in related academic work. Risk was measured with respect to a benchmark wealth level, which can be interpreted as a liabilitity level in case of Asset/Liability Management (ALM) problems. Using analytic Stochastic Programming (SP) techniques, we were able to formulate the optimal solutions in feedback form, i.e., as decision rules. This form has a clear-cut economic interpretation, which constitutes a valuable addition to the existing literature where solutions are often derived numerically rather than analytically.

One of the salient findings of the present paper is that the optimal decision rule is both non-linear and non-monotonic in either the surplus (assets minus discounted benchmark level) or the funding (wealth-to-benchmark) ratio. The amount invested in the risky asset is piece-wise linear in the surplus, and piece-wise non-linear (convexo-concave) in the funding ratio. Surprisingly, this result does not hinge on the distribution of the risky asset. The distribution only determines the relative steepness of the decision rule for positive and negative values of the surplus.

In contrast to many traditional continuous-time analyses involving utility functions, our model results in additional risk taking behavior both in situations of under-funding and over-funding. In case of under-funding, taking risk is the only way in which the 
probability on a positive final surplus can be made positive. In case of over-funding (i.e., a positive surplus), making risky investments increases expected return without unduely affecting downside-risk measures.

We also gave some practical guidelines concerning the return/downside-risk trade-off to be made in the model. By linking the model parameters to practical concepts like Valueat-Risk and recovery probabilities, we were able to simultaneously enhance managerial interpretability and reduce computational complexity.

The multi-stage version of our model results in "almost" myopic decisions. The model can be solved as a sequence of one-period models and the typical shape of the decision rules is constant over time. Its steepness, however, increases because the appropriate risk aversion parameter decreases over time. The decrease is brought about by the possible future recourse actions and leads directly to the paradigm of time diversification: asset allocations are riskier if the planning period is longer.

On the computational side, our analytic results also give rise to some interesting directions for future research. As the piece-wise linear structure of the optimal decision rules does not depend on the initial level of surplus nor on the precise form of the return distribution, optimal solutions to dynamic versions of our model can be found by an easy decomposition of the dynamic model in repeated one-period models. Such a decomposition allows for a considerable reduction in computational burden without affecting the optimality of the solution found.

Our results also have direct implications for more elaborate empirical financial planning models. Such models typically produce optimal solutions in the form of a decision (e.g., asset allocation or contribution policy) at each point in time and in every state of nature. This results in a large set of numbers that is often difficult to interpret and analyze. Presenting the optimal solutions in terms of decision rules that depend on key financial variables appears a good strategy. 


\section{Appendix: Proofs}

\section{Proof of Lemma 2.1:}

If $W_{0}=W_{0}^{B}$ the objective function simplifies to

$$
\max _{X_{0}} W_{0} r_{f}+X_{0} \cdot \mathbb{E}_{G}\left[u-r_{f}\right]-\lambda \cdot \mathbb{E}_{G}\left[-X_{0} \cdot\left(u-r_{f}\right)\right]^{+} .
$$

Clearly, as $\lambda>0$, if $X_{0}^{*} \leq 0$ then it is optimal to set $X_{0}^{*}=0$.

If $X_{0}^{*} \geq 0$ then (A1) is equal to

$$
W_{0} r_{f}+X_{0}^{*} \cdot \mathbb{E}_{G}\left[u-r_{f}\right]-\lambda X_{0}^{*} \cdot \mathbb{E}_{G}\left[r_{f}-u\right]^{+}
$$

If condition (3) does not hold, (A2) is maximized for $X_{0}^{*}=\infty$. Otherwise, $X_{0}^{*}=0$.

Proof of Lemma 2.2:

Substituting (1) in (2), we define the value of the objective function (2) for a certain $X_{0}$ by $F\left(X_{0}\right)$. Then for a fixed $X_{0}$

$$
F\left(X_{0}\right)=\mathbb{E}_{G}\left[W_{0} r_{f}+X_{0}\left(u-r_{f}\right)\right]-\lambda \cdot \int_{0}^{\bar{u}}\left(W_{1}^{B}-W_{0} r_{f}-X_{0}\left(u-r_{f}\right)\right) d G,
$$

where $G$ is the distribution function of $u$ and $\bar{u}$ is defined as

$$
\bar{u}=r_{f}+\frac{W_{1}^{B}-W_{0} r_{f}}{X_{0}}
$$

Differentiating (A3) with respect to $X_{0}$ gives the first and second order conditions

$$
\begin{aligned}
\frac{\partial F}{\partial X_{0}} & =\mathbb{E}_{G}\left[u-r_{f}\right]-\lambda \cdot \int_{0}^{\bar{u}}\left(r_{f}-u\right) d G=0, \\
\frac{\partial^{2} F}{\partial X_{0}^{2}} & =-\lambda \cdot \frac{\left(W_{1}^{B}-W_{0} r_{f}\right)^{2}}{X_{0}^{3}} \cdot g\left(\bar{u}\left(X_{0}\right)\right)<0,
\end{aligned}
$$

respectively. As $\lambda>0$, from (A6) it follows that $X_{0}$ is positive at the optimum.

\section{Proof of Theorem 2.1:}

Consider the value of the integral in (A5), see also Figure 1. As the integrand $r_{f}-u$ is positive for $0<\bar{u}<r_{f}$, it is clear that (A3) is increasing in $\bar{u}$ for $\bar{u}<r_{f}$. Similarly, (A3) decreases in $\bar{u}$ for $\bar{u}>r_{f}$. Using condition (3) and the fact that the expectation of the risky asset is larger than $r_{f}$, it follows that $F^{\prime}\left(X_{0}\right)=0$ solves for two distinct values $\bar{u}, \bar{u}^{(1)}<r_{f}$ and $\bar{u}^{(2)}>r_{f}$, corresponding to the two points of intersection of the integral as a function of $\bar{u}$ with the horizontal line $\mathbb{E}\left[u-r_{f}\right] / \lambda$, as in Figure 1. Note that $\bar{u}^{(1)}$ and $\bar{u}^{(2)}$ do not depend on $X_{0}$. Inverting (A4), we obtain the optimal decision

$$
X_{0}^{*}=\frac{W_{1}^{B}-W_{0} r_{f}}{\bar{u}^{(i)}-r_{f}},
$$


for $i$ either equal to 1 or 2 . From Lemma 2.2, $X_{0}^{*}>0$. Therefore, $\bar{u}^{(1)}<r_{f}$ can only be optimal if $W_{1}^{B}-W_{0} r_{f}<0$, i.e., there is a positive surplus $W_{0}-W_{0}^{B}$. Otherwise, $X_{0}^{*}$ would be negative. Also, $\bar{u}^{(2)}$ can only be optimal if $W_{1}^{B}-W_{0} r_{f}>0$. As the $\bar{u}^{(i)}$ correspond to a positive and a negative surplus, respectively, we can write $\bar{u}^{+}$for $\bar{u}^{(1)}$ and $\bar{u}^{-}$for $\bar{u}^{(2)}$. This gives the desired result as

$$
X_{0}^{*}= \begin{cases}\frac{r_{f}}{r_{f}-\bar{u}^{+}} \cdot\left(W_{0}^{B}-W_{0}\right) & \text { if } W_{0}>W_{0}^{B}, \\ \frac{r_{f}}{\bar{u}-r_{f}} \cdot\left(W_{0}^{B}-W_{0}\right) & \text { if } W_{0}<W_{0}^{B} .\end{cases}
$$

\section{Proof of Theorem 3.1:}

The first order condition for $X_{n-1}$ follows directly from Lemma 2.2 and is

$$
\int_{0}^{\bar{u}_{n}}\left(r_{f}-u_{n}\right) d G_{n}\left(u_{n}\right)-\mathbb{E}\left[u_{i}-r_{f}\right] / \lambda=0
$$

where $\bar{u}_{n}$ is defined as in (12). Clearly, this is consistent with expression (11) with $p_{n}^{-}=1$ and $p_{n}^{+}=0$.

To prove the theorem for $i<n$ we introduce the following sequence of value functions for the optimization problem in (9),

$$
\begin{aligned}
V_{i}\left(W_{i-1}\right) & =\max _{X_{i-1}} \mathbb{E}_{i-1}\left[V_{i+1}\left(W_{i-1} r_{f}+X_{i-1}\left(u_{i}-r_{f}\right)\right)\right], \quad i=1, \ldots, n, \\
V_{n+1}\left(W_{n}\right) & =W_{n}-\lambda \cdot\left[W_{n}^{B}-W_{n}\right]^{+}
\end{aligned}
$$

where $\mathbb{E}_{i-1}$ is defined as the conditional expectation given $u_{i-1}, u_{i-2}, \ldots, u_{1}$. Using (A10), the first order condition with respect to $X_{i-1}$ is

$$
\mathbb{E}_{i-1}\left[\frac{\partial V_{i+1}\left(W_{i}\right)}{\partial X_{i-1}}\right]=\mathbb{E}_{i-1}\left[\frac{\partial V_{i+1}\left(W_{i}\right)}{\partial W_{i}} \cdot \frac{\partial W_{i}}{\partial X_{i-1}}\right]=0,
$$

where $\partial W_{i} / \partial X_{i-1}=u_{i}-r_{f}$. Let $W_{i+1}^{*}=r_{f} W_{i}+X_{i}^{*}\left(W_{i}\right) \cdot\left(u_{i+1}-r_{f}\right)$, with $X_{i}^{*}\left(W_{i}\right)$ the optimal decision at time $i$. We have

$$
\begin{aligned}
\frac{\partial V_{i+1}\left(W_{i}\right)}{\partial W_{i}} & =\mathbb{E}_{i}\left[\frac{\partial V_{i+2}\left(W_{i+1}\right)}{\partial W_{i+1}} \cdot \frac{\partial W_{i+1}^{*}}{\partial W_{i}}\right]=r_{f} \cdot \mathbb{E}_{i}\left[\frac{\partial V_{i+2}\left(W_{i+1}\right)}{\partial W_{i+1}}\right] \\
& =\ldots=r_{f}^{n-i} \mathbb{E}_{i}\left[1-\lambda \cdot I_{\left\{S_{n}<0\right\}}\right]
\end{aligned}
$$

where $I_{A}$ is the indicator function of the event $A$. In this case it is equal to 1 if $S_{n}<0$ and 0 otherwise.

Using (A13), (A12) becomes

$$
\mathbb{E}_{i-1}\left[\frac{\partial V_{i+1}\left(W_{i}\right)}{\partial X_{i-1}}\right]=\mathbb{E}_{i-1}\left[r_{f}^{n-i}\left(u_{i}-r_{f}\right)\right]-\lambda \cdot \mathbb{E}_{i-1}\left[r_{f}^{n-i}\left(r_{f}-u_{i}\right) I_{\left\{S_{n}<0\right\}}\right]=0 .
$$

Partitioning based on the sign of $S_{i}$ gives

$$
\mathbb{E}_{i-1}\left[\left(r_{f}-u_{i}\right) I_{\left\{S_{n}<0\right\} \cap\left\{S_{i}<0\right\}}\right]+\mathbb{E}_{i-1}\left[\left(r_{f}-u_{i}\right) I_{\left\{S_{n}<0\right\} \cap\left\{S_{i}>0\right\}}\right]-\mathbb{E}_{i-1}\left[\left(u_{i}-r_{f}\right)\right] / \lambda=0 .
$$


Note that for $W_{i-1}$ given, $X_{i-1}^{*}$ is fixed and as the $u_{i}$ are independent, $S_{i}$ only varies monotonically with the realization of $u_{i}$. By absolute continuity of $G_{i}(\cdot)$, we do not have to consider the case $S_{i}=0$, so we can define a $\bar{u}_{i}$ such that $S_{i}>0$ for $u_{i}>\bar{u}_{i}$ and $S_{i}<0$ for $u_{i}<\bar{u}_{i}$. This reduces (A15) to

$$
\begin{gathered}
\int_{0}^{\bar{u}_{i}}\left(r_{f}-u_{i}\right) \operatorname{Pr}\left(S_{n}<0 \mid S_{i}\right) d G_{i}\left(u_{i}\right)+\int_{\bar{u}_{i}}^{\infty}\left(r_{f}-u_{i}\right) \operatorname{Pr}\left(S_{n}<0 \mid S_{i}\right) d G_{i}\left(u_{i}\right) \\
-\mathbb{E}\left[u_{i}-r_{f}\right] / \lambda=0
\end{gathered}
$$

Assume

$$
\operatorname{Pr}\left(S_{n}<0 \mid S_{i}\right)= \begin{cases}p_{i}^{+} & \text {if } S_{i}>0 \\ p_{i}^{-} & \text {if } S_{i}<0 .\end{cases}
$$

The above assumption states that the probability of ending up with a negative terminal surplus only depends on the sign of the time $i$ surplus $S_{i}$ and not on its the value. This clearly holds for $i=n$ with $p_{n}^{+}=0$ and $p_{n}^{-}=1$. This implies that equation (A16) can be rewritten as

$$
0=p_{i}^{-} \cdot \int_{0}^{\bar{u}_{i}}\left(r_{f}-u_{i}\right) d G_{i}\left(u_{i}\right)+p_{i}^{+} \cdot \int_{\bar{u}_{i}}^{\infty}\left(r_{f}-u_{i}\right) d G_{i}\left(u_{i}\right)-\mathbb{E}\left[u_{i}-r_{f}\right] / \lambda
$$

which simplifies to

$$
\int_{0}^{\bar{u}_{i}}\left(r_{f}-u_{i}\right) d G_{i}=\frac{\lambda^{-1}+p_{i}^{+}}{p_{i}^{-}-p_{i}^{+}} \cdot \mathbb{E}\left[u_{i}-r_{f}\right] .
$$

Parallel to the analysis for the one-period model, if condition (17) holds this equation solves for two distinct $\bar{u}_{i}$, depending on the sign of the surplus $S_{i-1}$ only. Moreover, the corresponding optimal $X_{i-1}$ is such that the next period's surplus is positive for $u_{i}>\bar{u}_{i}$ and negative for $u_{i}<\bar{u}_{i}$. Therefore,

$$
\begin{aligned}
\operatorname{Pr}\left(S_{n}<0 \mid S_{i-1}\right) & =\operatorname{Pr}\left(S_{n}<0 \mid S_{i}<0\right) \cdot \operatorname{Pr}\left(S_{i}<0 \mid S_{i-1}\right) \\
& +\operatorname{Pr}\left(S_{n}<0 \mid S_{i}>0\right) \cdot \operatorname{Pr}\left(S_{i}>0 \mid S_{i-1}\right) \\
& =p_{i}^{-} \cdot \int_{0}^{\bar{u}_{i}} d G_{i}\left(u_{i}\right)+p_{i}^{+} \cdot \int_{\bar{u}_{i}}^{\infty} d G_{i}\left(u_{i}\right),
\end{aligned}
$$

where $\bar{u}_{i}=\bar{u}_{i}^{+}$for $S_{i-1}>0$ and $\bar{u}_{i}=\bar{u}_{i}^{-}$for $S_{i-1}<0$. As $\bar{u}_{i}^{+}$and $\bar{u}_{i}^{-}$are constant, (A21) clearly shows that $\operatorname{Pr}\left(S_{n}<0 \mid S_{i-1}\right)$ also satisfies (A17). The proof now follows by induction.

\section{Proof of Corollary 3.1:}

The first order condition (15) is exactly the same as in the one-period model, except that $\lambda$ is replaced by $\lambda_{i}$. The result follows directly from applying Theorem 2.1.

\section{Proof of Corollary 3.2:}

Define $G_{i}^{-}$and $G_{i}^{+}$by $G_{i}\left(\bar{u}_{i}^{-}\right)$and $G_{i}\left(\bar{u}_{i}^{+}\right)$, respectively. From Theorem 3.1 it is clear that $\bar{u}_{i}^{-}>\bar{u}_{i}^{+}$, such that $G_{i}^{-}>G_{i}^{+}$for any $i$. 
Using (13) and (14), we can write

$$
p_{i}^{-}-p_{i}^{+}=\left(p_{i+1}^{-}-p_{i+1}^{+}\right) \cdot\left(G_{i+1}^{-}-G_{i+1}^{+}\right), \quad i=0, \ldots, n-1 .
$$

As $p_{n}^{-}>p_{n}^{+}$and $\left(G_{i+1}^{-}-G_{i+1}^{+}\right)<1$ for all $i$, this clearly implies that

$$
p_{i}^{-}-p_{i}^{+}<p_{i+1}^{-}-p_{i+1}^{+}, \quad i=0, \ldots, n-1,
$$

i.e., $\left\{p_{i}^{-}-p_{i}^{+}\right\}_{0}^{n}$ is a strictly positive and increasing sequence in $i$. Moreover, as $p_{i}^{-}>p_{i}^{+}$, it follows from (14) that $p_{i}^{+}>p_{i+1}^{+}$. Using this and (A23), it is easy to see that the numerator in (16) is decreasing in $\mathrm{i}$, while the denominator is increasing in $i$. Hence $\lambda_{i}^{-1}$ is decreasing in $i$, and thus increasing in the time to maturity $n-i$.

$$
\frac{p_{i}^{+}}{p_{i+1}^{+}}=\frac{p_{i+1}^{-}}{p_{i+1}^{+}} \cdot G_{i+1}^{+}+\left(1-G_{i+1}^{+}\right)>1, \quad i=0, \ldots, n-1,
$$

\section{Proof of Corollary 3.3:}

The rewritten first order condition in (15) shows that the first order condition for any $X_{i}$ has the same structure as in the one-period model. In the one-period model, the value of $\lambda$ determines the values $\bar{u}_{1}^{-}$and $\bar{u}_{1}^{+}$. As can be observed from Figure 1, a larger value of $\lambda$ lowers the horizontal line at level $\mathbb{E}\left[u-r_{f}\right] / \lambda$. As the $\lambda_{i}^{-1}$ are the equivalent of $\lambda^{-1}$ in the one-period model and the $u_{i}$ are identically distributed, Corollary 3.2 implies that the distance $\left|\bar{u}_{i}^{*}-r_{f}\right|$ is increasing in $i$. Hence, the absolute slope $r_{f} /\left|\bar{u}_{i}^{*}-r_{f}\right|$ is decreasing in $i$.

\section{Proof of Corollary 3.4:}

Define $G_{i}^{+}=G_{i}\left(\bar{u}_{i}^{+}\right)$and $G_{i}^{-}=G_{i}\left(\bar{u}_{i}^{-}\right)$. Given a value of $\lambda$, for the decision problem at time $n-1 \bar{u}_{n}^{+}$and $\bar{u}_{n}^{-}$are uniquely determined. They determine $G_{n}^{+}$and $G_{n}^{-}$, which determine $p_{n-1}^{+}$and $p_{n-1}^{-}$. Repeating this procedure for all previous subproblems finally gives a unique $p_{0}^{+}$or $p_{0}^{-}$, depending on whether $S_{0}>0$ or $S_{0}<0$, respectively. This implies that if a certain $p_{0}^{+}$or $p_{0}^{-}$is chosen, there will be either no feasible solution, or a unique $\lambda$ associated with it.

\section{References}

Artzner, P., F. Delbaen, J.-M. Eber, and D. Heath (1999). Coherent measures of risk. Mathematical Finance 9, 203-228.

Basak, S. and A. Shapiro (1999, June). Value-at-risk based risk management: Optimal policies and asset prices. Working paper, The Wharton School, University of Pennsylvania. 
Berger, A. J. and J. M. Mulvey (1998). The home account advisor, asset and liability management for individual investors. In W. Ziemba and J. M. Mulvey (Eds.), Worldwide Asset and Liability Modeling, pp. 634-665. Cambridge University Press.

Boender, C. G. E. (1997). A hybrid simulation/optimisation scenario model for asset/liability management. European Journal of Operational Research 99, 126-135.

Cariño, D., T. Kent, D. Myers, C. Stacy, M. Sylvanus, A. Turner, K. Watanabe, and W. Ziemba (1994, January-February). The russell-yasuda kasai model: An asset/liability model for a japanese insurance company using multistage stochastic programming. Interfaces 24, 29-49.

Consigli, G. and M. A. Dempster (1998). The CALM stochastic programming model for dynamic asset-liability management. Annals of Operations Research.

Dert, C. L. (1998). A dynamic model for asset liability management for defined benefit pension funds. In W. T. Ziemba and J. M. Mulvey (Eds.), Worldwide Asset Liability Modeling. Cambridge University Press.

Dupačová, J., M. Bertocchi, and V. Moriggia (1998). Postoptimality for scenario based financial planning models with an application to bond portfolio management. In W. T. Ziemba and J. M. Mulvey (Eds.), Worldwide Asset Liability Modeling, Chapter 12, pp. 263-285. Cambridge University Press.

Fishburn, P. C. (1977, March). Mean-risk analysis with risk associated with below-target returns. The american economic review 67(2), 116-126.

Hiller, R. S. and J. Eckstein (1993, November). Stochastic dedication: Designing fixed income portfolios using massively parallel benders decomposition. Management Science 39(11), 1422-1438.

Ingersoll, J. E. (1987). Theory of Financial Decision Making. Rowman \& Littlefield Publishers, Inc.

Kahneman, D. and A. Tversky (1979, March). Prospect theory: An analysis of decision under risk. Econometrica 47, 263-291.

Kritzman, M. and D. Rich (1998, Summer). Beware of dogma: The truth about time diversification. The Journal of Portfolio Management, 66-77.

Maranas, C. D., I. P. Androulakis, C. A. Floudas, A. J. Berger, and J. M. Mulvey (1997). Solving long-term financial planning problems via global optimization. Journal of Economics dynamics and control 21, 1405-1425.

Merton, R. C. (1969, August). Lifetime portfolio selection under uncertainty: The continuous-time case. Review of Economics and statistics 51, 247-257.

Merton, R. C. and P. A. Samuelson (1974). Fallacy of the log-normal approximation to optimal portfolio decision-making over many periods. Journal of Financial Economics 1, 67-94. 
Mulvey, J. M. and A. E. Thorlacius (1998). The towers perrin global capital market scenario generation system. In J. M. Mulvey and W. T. Ziemba (Eds.), Worldwide Asset and Liability Modeling, pp. 286-312. Cambridge University Press.

Samuelson, P. A. (1994, Fall). The long-term case for equities, and how it can be oversold. The Journal of Portfolio Management, 15-24.

Sethi, S. P. (1998). Optimal consumption-investment decisions allowing for bankruptcy: A survey. In W. T. Ziemba and J. M. Mulvey (Eds.), Worldwide Asset and Liability Modeling. Cambridge University Press.

Sortino, F. and R. Van der Meer (1991, Summer). Downside risk: Capturing what's at stake in investment situations. The Journal of Portfolio Management, 27-31.

Uryasev, S. and R. Rockafellar (1999). Optimization of conditional value-at-risk. Research report 99-4, University of Florida, Dept. of Industrial and Systems Engineering.

Ziemba, W. and J. Mulvey (Eds.) (1998). Worldwide Asset and Liability Modeling. Cambridge University Press. 